\title{
Análise de Estudos sobre Atenção Publicados em Periódicos Brasileiros
}

\author{
Patrícia Maria Uchôa Simões \\ Fundação Joaquim Nabuco - PE
}

\section{Resumo}

A atenção esteve presente nos estudos da Psicologia desde os introspeccionistas até a atualidade. Com a hegemonia do behaviorismo os estudos sobre atenção foram abandonados, e só no final da década de 50 e início de 60 houve uma retomada dessas questões, com o surgimento das ciências cognitivas. O presente estudo realizou uma pesquisa bibliográfica sobre o tema em periódicos brasileiros publicados entre 1999 e 2008 , encontrados na base de dados do SCIELO. Foram analisados 96 artigos identificados a partir do descritor atenção para palavras-chave. A análise incidiu sobre os aspectos: ano da publicação, área do periódico e palavras-chave. Os resultados mostraram um maior número de artigos na área da Saúde, com foco na hiperatividade infantil. A análise desses resultados propõe uma discussão sobre o papel que a atenção vem ocupando nos estudos em Psicologia e sugere uma agenda de pesquisas que supere a fragmentação das análises nos estudos.

Palavras-chave: atenção; consciência; psicologia.

\section{Review of Studies on Attention Published in Brazilian Journals}

\begin{abstract}
Attention has been a theme of Psychology studies since the introspectionist scholars until today. While the Behaviorism was a hegemonic approach, the studies of attention and consciousness have been abandoned. Only in the late 50 s and early $60 \mathrm{~s}$, with the emergence of cognitive science, these issues reemerged. In this study we developed a biliographical research on the subject, published in Brazilian journals between 1999 and 2008 , in the database SCIELO. The analysis identified 96 articles and focused on the aspects: the year of publication, the journal area and keywords. The results show a great number of articles in the area of Health, with a focus on child hyperactivity. The analysis of these results suggests a discussion on the role that has been occupying by the attention studies in Psychology and suggests a research agenda to overcome the fragmentation of the analyzes in the area. Keywords: Attention; conscience; psychology.
\end{abstract}

\section{Análisis de estudios sobre atención publicados en periódicos brasileños}

\section{Resumen}

La atención estuvo presente en los estudios de Psicología desde los introspeccionistas hasta los días actuales. Con la hegemonía del behaviorismo los estudios sobre atención y conciencia fueron abandonados y apenas al final de la década de 50 e inicio de la década de 60 , con el surgimiento de las ciencias cognitivas, estos temas fueron retomados. El presente estudio realizó una investigación bibliográfica sobre el tema en periódicos brasileños publicados entre 1999 y 2008 encontrados en la base de datos de SCIELO. Se analizaron 96 artículos identificados a partir del descriptor atención para palabras clave. El análisis incidió sobre tres aspectos: año de la publicación, área del periódico y palabras clave. El análisis de los resultados proponen discusión sobre el papel que la atención viene ocupando en los estudios en Psicología.

Palabras Clave: atención; conciencia; psicología. 


\section{Introdução}

A atenção sempre despertou grande interesse nos estudos em Psicologia, pelo seu papel na preparação e regulação das ações sensório-motoras e pela função subjacente que exerce no desenvolvimento de qualquer atividade cognitiva. Esse lugar privilegiado é explicado também pelas contribuições que o tema pode trazer para várias outras áreas, como educação, comunicação e marketing, bem como para o desenvolvimento de novas tecnologias da informação. Além desses pontos, nos dias atuais o interesse nessa área deve-se ainda ao crescente número de problemas de atenção detectados nas escolas, nos ambientes de trabalho, nas clínicas e nas relações interpessoais.

Todo esse interesse está registrado nas publicações relacionadas com essa temática que tratam de modelos teóricos que procuram explicar as formas e as relações da atenção com outras temáticas da Psicologia, especialmente a percepção, a memória e a aprendizagem, e com os estudos de intervenção que buscam solucionar problemas da atenção como hiperatividade, desatenção e impulsividade.

Vive-se hoje numa sociedade onde, desde a infância, a exposição excessiva à informação no cotidiano dos indivíduos impede a manutenção do foco de atenção, devido à velocidade e à multiplicidade com que as informações invadem o curso das atividades desenvolvidas durante o dia. Por um lado, a função básica de filtro de informações importantes para o desenvolvimento das atividades cotidianas é prejudicada pelo grande número de informações irrelevantes que chegam aos nossos sentidos; por outro, a função de atenção flutuante, responsável pelo pensamento criativo e divergente, não é valorizada numa sociedade que demanda a focalização e a distribuição dos recursos de atenção numa única direção, a qual é determinada pela produção e pelo sucesso profissional.

$\mathrm{Na}$ escola, registra-se de forma cada vez mais frequente o diagnóstico de problemas de atenção. Esse diagnóstico, baseado nos sintomas de hiperatividade, impulsividade e desatenção, associa-se a problemas de aprendizagem e de desempenho escolar (Bourjoun, \& Quaireau, 2000). As dificuldades com os mecanismos de atenção interferem na compreensão de textos, na resolução de problemas, no planejamento do estudo e na interação entre colegas e entre aluno e professor. Tudo isso atinge a autocofiança e a autoestima do aluno.

Não obstante, estudos na área apontam dificuldades no diagnóstico e a falta de consenso entre pesquisadores e profissionais quanto aos instrumentos e medidas que devem ser utilizados para avaliar a capacidade da atenção (Benczik, \& Casella, 2007). A diversidade de testes e abordagens metodológicas para o diagnóstico dificulta a comparação dos resultados obtidos nos estudos de avaliação da atenção. Quanto aos estudos de intervenção, a multiplicidade de abordagens teóricas e metodológicas tem como resultado diferentes propostas de tratamento, que vão desde a farmacoterapia até a intervenções psicoeducacionais e a abordagens psicoterapêuticas com a família.

\section{As funções da atenção}

Entre os estudos da área, diferentes funções da atenção foram investigadas na tentativa de delimitar e explicar os seus significados em diferentes contextos. Apesar de não se colocarem como contraditórios, esses estudos apresentam diferentes ênfases em suas formulações que nos permitem a compreender a complexidade de um mesmo fenômeno.

Essas diferenças entre as perspectivas focalizam a função seletiva de informações, ao enfatizar a limitação do processamento de informações (Sternberg, 2000) enquanto outras ressaltam a capacidade de distribuição dos recursos cognitivos para a realização simultânea de diferentes tarefas (Anderson, 2004). Ainda há autores que, mencionando tanto a função seletiva quanto a distributiva da atenção, buscam integrar numa mesma conceituação as duas funções da atenção (Boujon, \& Quaireau, 2000; Matlin, 2004); mas entre esses autores há consenso quanto à complexidade no envolvimento das funções da atenção e às formas diferentes de processamento em cada situação.

Observa-se também que, algumas definições incidem na relação entre atenção e outras funções cognitivas, como a percepção (Anderson, 2004; Matlin, 2004; Milner, 1978; Ratey, 2002; Schiffman, 2005; Sternberg, 2000), a memória (Boujon, \& Quaireau, 2000; Helene, \& Xavier, 2003; Sternberg, 2000), a aprendizagem (Boujon, \& Quaireau, 2000; Kastrup, 2004) e a consciência (Sternberg, 2000).

Os estudos que relacionam atenção e percepção focalizam a autorregulação porque é necessário limitar as informações que chegam através da percepção do corpo e do ambiente para que seja possível monitorar as próprias ações. Sendo assim, esses estudos têm especial interesse na função seletiva da atenção (Schiffman, 2005).

Quando relacionada à memória, é ressaltado o papel da atenção na codificação, armazenamento e resgate da informação. A forma de utilizar os recursos de atenção interfere no nível de profundidade com que a informação é codificada e na atribuição de significados àquilo que vai ser armazenado. Os níveis mais profundos de processamento e o armazenamento de conteúdos significativos resultam em códigos de memória mais duráveis (Sternberg, 2000).

Quanto aos estudos sobre atenção e aprendizagem, discute-se o processo de construção e integração das ideias do texto, no sentido de selecionar as principais, relacioná-las entre si e com outras ideias de fora do texto, para a formação de uma representação mental do texto (Kintsch, 1998).

Outros autores ainda discutem a intencionalidade na seleção da informação, ou seja, o caráter voluntário ou arbitrário da atenção. Essa discussão envolve a distinção entre processos automáticos e processos controlados (Matlin, 2004; Sternberg, 2000). Os primeiros ocorrem em níveis relativamente baixos de processamento cognitivo, envolvendo pouca ou nenhuma intenção ou esforço e geralmente fora do conhecimento consciente, e assim consomem poucos recursos de atenção. Essa forma de processar informações não requer controle ativo por parte do indivíduo, por isso pode ocorrer concomitantemente a outros processamentos e ser 
desencadeada por eventos do ambiente, sem a decisão voluntária do indivíduo e sem que este esteja prestando atenção à fonte da estimulação. Nesse tipo de processamento a atenção é denominada atenção automática ou atenção involuntária. Os processos controlados, por sua vez, exigem grande esforço mental e completo conhecimento consciente.

Diferentemente da forma de processamento anterior, esses processos demandam muitos recursos de atenção, o que não possibilita o desempenho concomitante de duas tarefas. Além disso, apresentam o controle voluntário da atenção e a decisão consciente do indivíduo em manter a atenção focalizada em uma determinada atividade. Neste segundo tipo de processamento a atenção é denominada atenção voluntária ou atenção arbitrária.

Esses aspectos destacados da discussão sobre o conceito de atenção apontam esse fenômeno como um mecanismo subjacente a todas as funções cognitivas, de forma que o desempenho em qualquer tipo de processamento ou de atividade depende do modo como é utilizado.

Compreende-se ainda que pode haver diferentes funções de atenção, dependendo do tipo de processamento envolvido na tarefa. A amplitude dessa definição envolve a grande dificuldade em estudar a temática. Kastrup (2007) utiliza um conceito bastante amplo, que parece envolver os aspectos aqui discutidos:

atenção, enquanto processo complexo, pode assumir diferentes funcionamentos: seletivo ou flutuante, focado ou desfocado, concentrado ou disperso, voluntário ou involuntário, em várias combinações como seleção voluntária, flutuação involuntária, concentração desfocada, focalização dispersa, etc. Embora as variedades atencionais coexistam de direito, elas ganham organizações e proporções distintas na configuração de diferentes políticas cognitivas (p. 15).

\section{Os estudos sobre atenção}

Como já mencionado anteriormente, apesar de na atualidade haver grande aumento da difusão dos estudos sobre atenção e, especialmente, sobre os problemas de atenção, esta temática esteve presente desde o início dos estudos da Psicologia. Em 1890, Willian James definiu a atenção como sendo constituída pela focalização, concentração e consciência. Esse pioneiro entre os pesquisadores da cognição investigou a quantidade de ideias que um indivíduo pode ter ao mesmo tempo (tema que ainda está presente entre os estudos atuais) e classificou os modos de atenção em: a) ativos, quando controlados pelos objetivos ou expectativas do indivíduo, e b) passivos, quando controlados por estímulos externos (Matlin, 2004).

Em 1911, ainda nos primeiros momentos de surgimento da Psicologia enquanto área da ciência, Wilheim Wundt denominou, do ponto de vista estrutural, campo subjetivo como a área no interior da qual estariam presentes conteúdos com graus variados de claridade ou nitidez. Os mais claros e distintos se encontrariam no entorno de um ponto de fixação presente no foco de atenção, compondo uma espécie de consciência clara, e aqueles que circundam tal foco e se distribuem indistintamente até a periferia do campo subjetivo formariam uma consciência obscura. Do ponto de vista funcional, definiu apreensão como o processo responsável pelo acesso àquelas percepções que se distribuiriam pelo campo subjetivo mais amplo, o da consciência obscura; e apercepção foi por ele definida como a atividade em que elementos presentes no campo da consciência obscura poderiam ser elevados, por meio da atenção, ao campo da consciência clara ou foco de atenção. Dessa forma, compreende processo aperceptivo como a atividade de tornar conscientes conteúdos antes inconscientes num caráter dinâmico e plástico (Matlin, 2004).

A partir desses primeiros estudos, as pesquisas sobre a atenção desenvolveram-se tendo como pressuposto que o campo de estudo da atenção e o campo de estudo da consciência fazem parte de um sistema unitário. Esse pressuposto levou muitos autores que desenvolviam estudos sobre a atenção a considerarem a consciência como seu objeto de investigação (Anderson, 2004; Matlin, 2004; Sternberg, 2000).

Com a hegemonia do behaviorismo entre as pesquisas no campo da Psicologia, os estudos sobre atenção e consciência foram abandonados, por fenômenos mentais não observáveis e imensuráveis. Apenas no final da década de 1950 e início da década de 1960, com o surgimento dos estudos nas ciências cognitivas, foram retomada essas questões e o interesse dos pesquisadores foi novamente direcionado para o estudo da atenção (Anderson, 2004; Matlin, 2004; Sternberg, 2000).

Nas últimas décadas, numa perspectiva cognitivista, os estudos da atenção utilizam dois modelos gerais, baseados: a) no processamento fisiológico da detecção de estímulos - processos bottom-up; b) nos processos superiores de memória e de representações mentais - processos top-down. Alguns autores consideram a atenção como uma forma de processamento intermediário que integra os dois processos mencionados (Schiffman, 2005; Sternberg, 2000).

\section{Os modelos teóricos da atenção}

Diferentes modelos teóricos foram propostos para explicar o mecanismo de atenção. Alguns autores, como Anderson (2004), Matlin (2004) e Sternberg (2000), organizam os estudos sobre esses modelos em dois grandes grupos de teorias.

Um primeiro grupo de teorias comparou o mecanismo de atenção com um gargalo ou com um filtro de informação entre todas as informações disponíveis no meio, a cada momento. Esses modelos da atenção propõem uma passagem estreita que controlaria e limitaria a quantidade de informações às quais podemos prestar atenção. Dessa forma, as pessoas seriam extremamente limitadas na quantidade de informações que podem processar num dado momento. 
Esse modelo foi proposto inicialmente por Broadbent (1958 conforme citado por Sternberg, 2000, p. 100), mas outras versões foram propostas por outros autores.

Esse primeiro grupo de teorias foi criticado por subestimar a flexibilidade da atenção humana $\mathrm{e}$ desconsiderar até que ponto o treinamento pode alterar a utilização da atenção (Matlin, 2004). Essas teorias parecem explicar satisfatoriamente situações que demandam uma atenção focalizada e seletiva, mas não ajudam na explicação da utilização da atenção em situações que demandem a distribuição da atenção. Procurando responder a essas críticas, e a partir de novas evidências empíricas, algumas reformulações foram propostas considerando-se que algumas informações são enfraquecidas e não filtradas com base em suas propriedades físicas. A limitação não estaria no sistema perceptivo, mas no sistema de respostas do indivíduo. Essas teorias são conhecidas como teorias da atenuação ou teorias da seleção tardia, e foram propostas em diversos estudos como, por exemplo, o de Treisman (1964 conforme citado por Sternberg, 2000, p. 101) e o de Deutsch e Deutsch (1963 conforme citado por Sternberg, 2000, p. 101).

Um segundo grupo de teorias, conhecidas como teorias modulares ou teorias do gargalo central, compara o cérebro com um computador, composto de alguns sistemas de processamento em paralelo para os diversos sistemas perceptivos, os sistemas motores e a cognição central. Cada um desses sistemas funcionaria como um subsistema especializado que tem seus próprios gargalos que focalizam o processamento em uma atividade. Essa compreensão da atenção explica a nossa capacidade de distribuir recursos atentivos em atividades diferentes quando as exigências das modalidades de atividades não são conflitantes, e assim podemos processar várias fontes de estímulo ao mesmo tempo. Nesse grupo se encontram os estudos de Cave e Wolfe
(1990 conforme citado por Sternberg, 2000, p. 103), Duncan e Humpheys (1989 conforme citado por Sternberg, 2000, p. 103) e Kahneman (1973 conforme citado por Sternberg, 2000, p. 102).

A crítica feita a esse último grupo de teorias é que essas teorias seriam excessivamente amplas e vagas e não ofereceriam elementos para a compreensão do processamento seletivo de informações; no entanto, parecem explicar melhor o desempenho em tarefas que exigem a atenção dividida, embora não expliquem todos os aspectos da atenção (Sternberg, 2000). Assim, esses modelos teóricos não parecem excludentes ao buscarem explicar os mecanismos de atenção, mas apresentam explicações parciais e até mesmo complementares desses mecanismos (Matlin, 2004; Sternberg, 2000).

Essas formas de processar informações estão presentes no uso dos recursos atentivos para finalidades diferentes. A demanda da atividade, em parte, determinaria o tipo de processamento utilizado.

\section{Os tipos de atenção}

Podem-se distinguir diferentes tipos ou funções da atenção que consideram o processamento cognitivo envolvido. Benczik \& Casella (2007) afirmam que "a atenção não pode ser considerada como uma função única de caráter global" (p.39).

Muitas denominações são encontradas na literatura da área. Na tentativa de sintetizar e sistematizar os estudos sobre as funções da atenção, foi construído o quadro abaixo, que lista as diferentes denominações ligando aquelas que parecem tratar do mesmo mecanismo atentivo (Benczik, \& Casella, 2007; Boujon, \& Quaireau, 2000; Matlin, 2004; Simões, \& Tiedeman, 1977; Sternberg, 2000):

QUADRO 1. Tipos de atenção e o processamento cognitivo envolvido.

\begin{tabular}{|c|c|}
\hline Tipos de atenção & Definição \\
\hline $\begin{array}{l}\text { vigilância (Simões, \& Tiedeman, 1977; Sternberg, 2000) } \\
\text { atenção mantida (Benczik, \& Casella, 2007) } \\
\text { atenção sustentada (Benczik, \& Casella, 2007) } \\
\text { atenção contínua (Boujon, \& Quaireau, 2000) }\end{array}$ & $\begin{array}{l}\text { estado de preparação para detectar e responder a } \\
\text { determinadas mudanças no ambiente }\end{array}$ \\
\hline sondagem (Sternberg, 2000) & procura ativa por um determinado estímulo \\
\hline $\begin{array}{l}\text { atenção seletiva (Benczik, \& Casella, 2007; Boujon, \& Quaireau, } \\
\text { 2000; Matlin, 2004; Simões, \& Tiedeman, 1977; Sternberg, 2000) } \\
\text { atenção focalizada (Boujon, \& Quaireau, 2000) } \\
\text { atenção focada (Benczik, \& Casella, 2007) }\end{array}$ & $\begin{array}{l}\text { escolha de um estímulo em relação a outro para } \\
\text { prestar atenção }\end{array}$ \\
\hline $\begin{array}{l}\text { atenção dividida (Benczik, \& Casella, 2007; Boujon, \& Quaireau, } \\
\text { 2000; Matlin, 2004; Simões, \& Tiedeman, 1977; Sternberg, 2000) }\end{array}$ & $\begin{array}{l}\text { distribuição dos recursos de atenção para coordenar o } \\
\text { desempenho em mais de uma tarefa }\end{array}$ \\
\hline $\begin{array}{l}\text { atenção conjunta (Boujon, \& Quaireau, 2000) } \\
\text { atenção compartilhada (Bosa, 2002) }\end{array}$ & $\begin{array}{l}\text { ajuste do olhar em uma direção, em resposta aos } \\
\text { deslocamentos do olhar do outro com quem interage }\end{array}$ \\
\hline atenção alternada (Benczik, \& Casella, 2007) & capacidade do individuo mudar o foco de atenção \\
\hline
\end{tabular}


A quantidade de denominações da atenção e de suas funções revela a complexidade do fenômeno da atenção e alerta quanto ao cuidado necessário para não fragmentar o seu estudo. As qualificações da atenção indicam as substantividades das funções, necessitando, então, de estudos que possam integrar as diferentes funções na explicação do mesmo fenômeno.

\section{II - Os objetivos e os procedimentos desse estudo}

Considerando os aspectos apresentados e discutidos relativos à atenção, o presente estudo teve como objetivo compreender as preocupações dos autores e os rumos que os estudos publicados em periódicos nacionais estão tomando na investigação sobre essa temática.

Tabela 1. Número de artigos por periódico.

\begin{tabular}{|c|c|}
\hline PERIÓDICOS & № de artigos \\
\hline Arquivos de Neuropsiquiatria & 32 \\
\hline Revista Brasileira de Psiquiatria & 13 \\
\hline Jornal Brasileiro de Psiquiatria & 10 \\
\hline Revista de Psiquiatria Clínica & 9 \\
\hline Psicologia \& Sociedade & 4 \\
\hline Psicologia: Teoria e Pesquisa & 4 \\
\hline Arquivos Brasileiros de Oftalmologia & 3 \\
\hline Revista Brasileira de Oftalmologia & 3 \\
\hline Revista CEFAC - Atualização Científica em Fonoaudiologia e Educação & 3 \\
\hline Revista de Psiquiatria (RS) & 3 \\
\hline Estudos de Psicologia (Natal) & 2 \\
\hline Revista da Escola de Enfermagem da USP & 2 \\
\hline Jornal de Pediatria (Rio) & 1 \\
\hline Paidéia & 1 \\
\hline Pro-Fono Revista de Atualização Cientíica & 1 \\
\hline Psicologia em Estudo & 1 \\
\hline Psicologia: Reflexão e Crítica & 1 \\
\hline Revista Brasileira de Fisioterapia & 1 \\
\hline Revista Brasileira de Saúde Materno Infantil & 1 \\
\hline Revista da Sociedade Brasileira de Fonoaudiologia & 1 \\
\hline
\end{tabular}


Tabela 2. Número de artigos por ano e área dos periódicos.

\begin{tabular}{l|l|l|l|l|l|l|l|l|l|l|l}
\hline \multirow{2}{*}{ ÁREA } & \multicolumn{9}{l}{ ANO DA PUBLICAÇÃO } \\
\cline { 2 - 25 } & 1999 & 2000 & 2001 & 2002 & 2003 & 2004 & 2005 & 2006 & 2007 & 2008 & Total \\
\hline Saúde & 2 & 1 & 6 & 2 & 8 & 9 & 10 & 8 & 24 & 13 & $83(86,5 \%)$ \\
\hline Humanas & 0 & 0 & 0 & 2 & 0 & 2 & 0 & 3 & 3 & 3 & $13(13,5 \%)$ \\
\hline Total & 2 & 1 & 6 & 4 & 8 & 11 & 10 & 11 & 27 & 16 & 96 \\
\hline
\end{tabular}

Pretende-se propor uma reflexão sobre o lugar que a Psicologia vem ocupando nessa investigação, que, além de estar presente historicamente entre os temas na Psicologia, reveste-se na atualidade de grande importância pela necessidade de respostas para o tratamento dos distúrbios a ela associados.

Para essa análise foi realizada uma pesquisa bibliográfica dos trabalhos sobre o tema publicados na base de dados Scielo Brazil - Scientific Eletronic Library Online, a partir da busca por assunto, utilizando-se como descritor a palavra-chave atenção.

Após a identificação dos artigos, a análise considerou o ano de publicação, a área do periódico onde foi publicado o artigo e as palavras-chave.

\section{III - Os resultados deste estudo}

Foram identificados 96 artigos publicados entre os anos de 1999 e 2008, em 20 periódicos diferentes, com a palavra-chave atenção. Foram excluídos os artigos nos quais o termo atenção não se referia a um processo cog-

Tabela 3. Número de artigos com palavras que indicam faixa etária.

\begin{tabular}{l|l|c}
\hline \multirow{2}{*}{ Faixa etária } & Palavras-chave & № de artigos \\
\hline \multirow{4}{*}{ Infância } & criança & 7 \\
\cline { 2 - 3 } & crianças & 2 \\
\cline { 2 - 3 } & desenvolvimento infantil & 1 \\
\cline { 2 - 3 } & infância & 1 \\
\hline \multirow{3}{*}{ Adolescência } & adolescente & 2 \\
\cline { 2 - 3 } & remissão na adolescência & 1 \\
\hline \multirow{2}{*}{ Adulta } & adulto & 4 \\
\cline { 2 - 3 } & adultos & 3 \\
\hline
\end{tabular}

nitivo, mas à ação de atender, atendimento (por exemplo, atendimento em saúde, atendimento clínico etc.).

A tabela 1 apresenta o número de artigos por periódico, ressaltando-se que mais de dois terços dos artigos foram publicados em periódicos da área específica da Psiquiatria.

Tabela 4. Número de artigos com palavras que indicam funções cognitivas

\begin{tabular}{l|c}
\hline Palavras-chave & № de artigos \\
\hline aprendizagem & 3 \\
\hline audição & 1 \\
\hline Cognição & 6 \\
\hline compreensão & 1 \\
\hline funções executivas & 1 \\
\hline Leitura & 2 \\
\hline linguagem & 1 \\
\hline Memória & 1 \\
\hline memória de trabalho & 1 \\
\hline memória explícita & 1 \\
\hline memória implícita & 1 \\
\hline memória operacional & 1 \\
\hline percepção auditiva & 1 \\
\hline percepção de movimento & 1 \\
\hline percepção visual & 1 \\
\hline procura visual & 1 \\
\hline recognição/invenção & 1 \\
\hline visão binocular/fisiologia & 1 \\
\hline TOTAL & 1 \\
\hline
\end{tabular}


Tabela 5. Número de artigos com palavras que se referem a problemas de atenção.

\begin{tabular}{|c|c|c|}
\hline Problemas de atenção & Palavras-chave & $\begin{array}{l}\text { № de } \\
\text { artigos }\end{array}$ \\
\hline \multirow{15}{*}{$\begin{array}{l}\text { Hiperatividade } \\
\text { (total 64) }\end{array}$} & déficit de atenção e hiperatividade & 2 \\
\hline & distúrbio de déficit de atenção e hiperatividade & 1 \\
\hline & distúrbio de hiperatividade com déficit de atenção & 1 \\
\hline & Hiperatividade & 6 \\
\hline & síndrome de hiperatividade com déficit de atenção & 1 \\
\hline & TDAH & 10 \\
\hline & transtorno da falta de atenção com hiperatividade & 8 \\
\hline & transtorno de déficit de atenção e hiperatividade & 3 \\
\hline & transtorno de déficit de atenção/hiperatividade & 3 \\
\hline & transtorno do déficit de atenção com hiperatividade & 1 \\
\hline & transtorno do deficit de atenção e hiperatividade & 14 \\
\hline & transtorno do deficit de atenção e hiperatividade (tdah) & 1 \\
\hline & transtorno do deficit de atenção/hiperatividade & 10 \\
\hline & transtorno hipercinético & 1 \\
\hline & transtornos da falta de atenção com hiperatividade & 2 \\
\hline \multirow{5}{*}{$\begin{array}{l}\text { Desatenção } \\
\text { (total 13) }\end{array}$} & déficit de atenção & 4 \\
\hline & distúrbio de déficit de atenção & 1 \\
\hline & transtorno de déficit de atenção & 2 \\
\hline & transtorno do deficit de atenção & 5 \\
\hline & Desatenção & 1 \\
\hline \multirow{2}{*}{$\begin{array}{l}\text { Impulsividade } \\
\text { (total 2) }\end{array}$} & déficit de atenção com hiperatividade/impusividade & 1 \\
\hline & Impulsividade & 1 \\
\hline \multicolumn{2}{|l|}{ Total } & 79 \\
\hline
\end{tabular}

Os artigos foram classificados quanto à área do periódico no qual se encontravam, seguindo a classificação do Scielo pelas áreas do conhecimento. A tabela 2 mostra as áreas dos periódicos pelo ano de publicação. Reafirmando o resultado já comentado na tabela anterior, foi encontrado um número muito superior de artigos publicados em periódicos da área da Saúde.

Também se observa um pequeno crescimento no número de publicações entre os anos investigados; no entanto, quando se trata de uma análise comparativa, observase que o aumento das publicações na área da Saúde foi bem maior que o ocorrido entre os estudos da área da Psicologia.

Foram identificadas 396 palavras-chave, considerando-se o total de artigos, e 238 palavras diferentes que não se repetiram. Dentre estas, 190 foram mencionadas apenas uma vez e 22 foram mencionadas duas vezes. Esses números apontam a diversidade das temáticas investigadas pelos estudos sobre atenção.

Foram encontradas palavras que indicam faixas etárias. Os quantitativos dessas palavras estão apresentados na tabela 3. Destaca-se o número de palavras relacionadas à infância, o que evidencia a preocupação maior com essa etapa em comparação com as outras, quando se trata da temática atenção.

As palavras relacionadas a funções cognitivas foram agrupadas na tabela 4 . Ressalta-se a variedade de funções cognitivas mencionadas, o que indica haver consenso em grande número de artigos quanto à importância da atenção no desempenho de diferentes funções cognitivas, mesmo sendo diferentes essas funções.

A tabela 5 apresenta o grupo de palavras que mencionam problemas, distúrbios e dificuldades relacionados à 
Tabela 6. Número de artigos com palavras relacionadas a comportamentos, condutas e psicopatologias

\begin{tabular}{|c|c|}
\hline Palavras-chave & № de artigos \\
\hline alcoolismo/dependência & 2 \\
\hline Ansiedade & 1 \\
\hline autismo/autismo infantil/ transtornos do espectro autístico & 3 \\
\hline bulimia/bulimia nervosa & 2 \\
\hline comportamento disruptivo & 1 \\
\hline comportamento impulsivo & 1 \\
\hline comportamento precorrente & 1 \\
\hline depressão/transtornos depressivos & 3 \\
\hline distúrbio de personalidade e comportamento & 1 \\
\hline distúrbios psicóticos & 1 \\
\hline doença de Alzheimer & 1 \\
\hline epilepsia parcial complexa & 1 \\
\hline esclerose múltipla & 2 \\
\hline síndrome de Asperger & 1 \\
\hline síndrome de Tourette & 1 \\
\hline TID & 2 \\
\hline transtorno bipolar & 1 \\
\hline transtorno da conduta & 1 \\
\hline transtorno de/do déficit da atenção e do comportamento disruptivo & 2 \\
\hline transtorno de oposição desafiante & 1 \\
\hline transtornos de pânico/farmacoterapia & 1 \\
\hline transtorno do comportamento infantil & 1 \\
\hline transtorno obssessivo-compulsivo & 1 \\
\hline transtorno opsitivo-desafiador & 1 \\
\hline transtornos alimentares/transtornos da alimentação & 2 \\
\hline transtornos cognitivos & 3 \\
\hline transtornos de déficit da atenção e do comportamento disruptivo & 1 \\
\hline transtornos de memória & 2 \\
\hline transtornos invasivos do desenvolvimento & 1 \\
\hline transtornos mentais da criança & 1 \\
\hline TOTAL & 43 \\
\hline
\end{tabular}

atenção. Destaca-se o número de ocorrências de palavraschave relacionadas à hiperatividade, indicando a maior preocupação dos estudos com essa dificuldade.

As palavras também foram agrupadas quanto à menção a comportamentos, condutas e/ou psicopatologias. A tabela 6 apresenta as palavras relacionadas nesse agrupamento. Aqui também se registra o grande número de ocor- rências, o que mostra a atribuição de relações entre atenção e uma grande diversidade de psicopatologias.

Um número expressivo de artigos continha palavras relacionadas a substâncias químicas medicamentosas. Essas palavras são apresentadas na tabela 7. Esses dados apontam a medicalização na discussão dos distúrbios de atenção. 
Tabela 7. Número de artigos com palavras relacionadas a substâncias químicas.

\begin{tabular}{l|l}
\hline Palavras-chave & № de artigos \\
\hline Amitripilina & 1 \\
\hline analgésicos opióides & 1 \\
\hline Antidepressivos & 1 \\
\hline bupropiona & 1 \\
\hline Galantamina & 1 \\
\hline Farmacoterapia & 1 \\
\hline metilfenidato & 3 \\
\hline nortriptilina/uso terapêutico & 1 \\
\hline Psicoestimulantes & 1 \\
\hline TOTAL & 11 \\
\hline
\end{tabular}

Tabela 8. Número de artigos com palavras relacionadas a tratamento, intervenção

\begin{tabular}{l|l}
\hline Palavras-chave & № de artigos \\
\hline farmacoterapia & 1 \\
\hline tratamento & 3 \\
\hline terapia cognitiva & 1 \\
\hline resultado de tratamento & 1 \\
\hline grupos psicoeducativos & 1 \\
\hline TOTAL & 7 \\
\hline
\end{tabular}

As palavras que indicam tratamento ou intervenção estão apresentadas na tabela 8 . O número pequeno de estudos que utilizaram esse grupo de palavras mostra a carência de pesquisas de intervenção ou que avaliem formas de condutas terapêuticas na área.

\section{IV - Discussão dos resultados}

Os resultados apresentados nesta pesquisa descrevem um quadro panorâmico da produção acadêmica no Brasil sobre o fenômeno da atenção. O crescente número de estudos sobre essa temática revela a importância que vem adquirindo o debate científico sobre a capacidade de utilizar os recursos cognitivos no que se refere à focalizar a atenção como a sua distribuição em diferentes atividades ao mesmo tempo.

Também se destaca o interesse dos estudos das áreas da Saúde, em especial da Psiquiatria, bem como o foco na psicopatologia infantil, quase sempre nomeando os problemas relacionados à capacidade atentiva de hiperatividade. Esses resultados estão de acordo com estudos anteriores que assumem perspectivas sociológicas no tratamento do tema e discutem as questões relativas á atenção em seu contexto histórico e social, como os estudos de Caliman (2008a, 2008b, 2009) e o estudo de Legnani e Almeida (2008).

Observou-se também, assim como no estudo de Benczik e Casella (2007), a falta de concordância entre os termos utilizados nas pesquisas e a diversidade destas pesquisas. Isto mostra quanto temos a investigar no sentido de construir acordos para comparações e debates entre posições e conhecimentos. Por outro lado, os resultados apontaram diferenças de abordagens que, se fossem consideradas, permitiriam o confronto de perspectivas, requisito necessário para o avanço do conhecimento. Da forma como vem acontecendo, como já alertaram Oliveira e Albuquerque (2009), o debate entre abordagens não ocorre e o conhecimento permanece estanque, sem que ocorra diálogo entre posições teóricas e epistemológicas sobre o tema.

Por fim, a quase escassez de estudos sobre o tratamento de pessoas com problemas de atenção que partam de uma abordagem psicológica e o número de estudos sobre tratamentos com medicalização questionam o que os psicólogos conseguiram acumular de conhecimentos sobre essa temática, que é histórica no desenvolvimento da Psicologia enquanto ciência. Os significados dessa omissão nos levam a buscar explicações sobre as tendências e rumos da pesquisa e da produção bibliográfica sobre o tema na área.

\section{V - As considerações finais desse estudo}

Pretende-se que a apresentação desses resultados leve a uma reflexão sobre o lugar da pesquisa sobre a atenção na Psicologia. A preocupação com o tema não tem levado a reflexões epistemológicas e as análises fragmentadas dificultam a compreensão do fenômeno como um todo e sua inserção no contexto social e histórico atual.

A preponderância dos estudos na área da saúde convida os investigadores da Psicologia a contribuir com o seu olhar e tratamento da questão e alerta para a abordagem medicamentosa presente na maioria dos estudos. As preocupações com os problemas de atenção e os aspectos desta nos quais esses problemas podem estar interferindo quanto ao desempenho cognitivo e às relações interpessoais exigem da Psicologia uma explicação que vá além do âmbito das ciências médicas. 
Uma agenda de pesquisas sobre a atenção dentro da Psicologia tem como desafio a discussão epistemológica do fenômeno e a busca de análises que rompam com a fragmentação dos estudos sobre o processo de atenção. Trata-se de um processo complexo e, para explicá-lo, faz-se necessário ir além de sua dimensão funcional e utilitária, a partir de modelos explicativos que, além de abarcar a psicodinâmica da utilização dos recursos atentivos nas atividades desenvolvidas no cotidiano, discutam uma concepção de indivíduo e de mundo. Assim, a Psicologia deve oferecer sua contribuição fornecendo elementos para a compreensão desse fenômeno e propondo estratégias de intervenção que possam auxiliar os indivíduos que apresentem dificuldades relativas à atenção no desempenho de suas atividades cotidianas

\section{Referências}

Anderson, J.R. (2004). Psicologia Cognitiva e suas implicações experimentais. Rio de Janeiro: LTC.

Benczic, E.B.P., \& Casella, E.B. (2007). Atenção. Em Schelini, P.W. (Org.) Alguns Domínios da Avaliação Psicológica (pp. 31-58). Campinas, SP: Alínea

Bosa, C. (2002). Atenção Compartilhada e identificação precoce do Autismo. Psicologia: Reflexão e Crítica, 15(1), 77-88.

Boujon, C., \& Quaireau, C. (2000). Atenção e aproveitamento escolar. São Paulo: Loyola.

Caliman, L.V. (2008a). O TDAH: entre as funções, disfunções e otimização da atenção. Psicologia em Estudo, 13(3), 559-566.

Caliman, L.V. (2008b). Os valores da atenção e a atenção como valor. Estudos e Pesquisas em Psicologia, 8 (3), 632-645.
Caliman, L.V. (2009). A constituição sócio-médica do "fato TDAH". Psicologia \& Sociedade, 21(1), 135-144.

Helene, A.F., \& Xavier, G.F. (2003). A Construção da Atenção a partir da Memória. Revista Brasileira de Psiquiatria, 25(Suppl. 2), 12-20.

Kastrup, V. (2004). A aprendizagem da Atenção na Cognição Inventiva. Psicologia \& Sociedade, 16 (3), 7-16.

Kastrup, V. (2007). O funcionamento da Atenção no trabalho do cartógrafo. Psicologia \& Sociedade, 19(1), 15-22.

Kintsch, W. (1998). Comprehension: A paradigm for cognition. Cambridge: University Press.

Legnani, V.N., \& Almeida, S.F. (2008). A construção diagnóstica de Transtorno de Déficit de Atenção/Hiperatividade: uma discussão crítica. Arquivos Brasileiros de Psicologia, 60 (1), 02-13.

Matlin, M.W. (2004). Psicologia Cognitiva. Rio de Janeiro: LTC.

Milner, P. (1978). Psicologia Fisiológica. São Paulo: Cultrix.

Oliveira, C.G., \& Albuquerque, P.B. (2009). Diversidade de resultados no estudo do transtorno de déficit de atenção e hiperatividade. Psicologia: Teoria e Pesquisa, 25(1), 93-102.

Ratey, J.J. (2002). O Cérebro: um guia para o usuário. Rio de Janeiro: Objetiva.

Schiffman, H.R. (2005). Sensação e Percepção. Rio de Janeiro: LTC.

Simões, E.A.Q., \& Tiedemann, K.B. (1977). Psicologia da Percepção. São Paulo: EPU.

Sternberg, R.J. (2000). Psicologia Cognitiva. Porto Alegre: ArtMed.

\section{Sobre a autora}

Patrícia Maria Uchôa Simões (pusimoes@gmail.com)

Fundação Joaquim Nabuco. Doutora em Psicologia Cognitiva

Trabalho apresentado na sessão coordenada com o título: Tópicos da Psicologia Cognitiva Contemporânea, na XXXIX Reunião Anual de Psicologia, em Goiânia 